Classification of Inpatients by Needs

\begin{tabular}{|c|c|c|}
\hline Staff/Patient Ratio & Facilities Required & Categories of Care \\
\hline High & High & Coronary care, poison unit \\
\hline Moderate & Medium & $\begin{array}{l}\text { Intensive care, renal dialysis } \\
\text { Routine acute care-for example, } \\
\text { geriatric assessment, elective } \\
\text { surgery, progressing seriously ill, } \\
\text { etc. }\end{array}$ \\
\hline Low & Low & $\begin{array}{l}\text { Five-day investigation units, } \\
\text { rehabilitation, convalescence }\end{array}$ \\
\hline Moderate & Low & $\begin{array}{l}\text { Day stay units-minor operations, } \\
\text { specialized acute investigation, } \\
\text { cytotoxic treatment, etc. }\end{array}$ \\
\hline
\end{tabular}

be distributed more efficiently and hence economically. Various categories may be identified (see table).

This sort of organization fits into a divisional system and is in operation, in part at least, in several hospitals. Districts served by several hospitals could benefit if divisional concepts developed among hospitals.

Consultants and registrars could be more energetic in their efforts to complete investigations in outpatients. Good clinic organization, with appropriate paramedical staffing, can probably reduce the burdens on the inpatient departments attributable to diabetes, hypertension, and chronic respiratory disease. Day-stay units, if used properly, can treat many patients at present managed in hospital. Extension of facilities in the home, and better liaison among all the groups concerned may yet avert the expected geriatric avalanche, as well as many other so-called acute admissions. Consultants could be used on a domiciliary basis rather more than appears to be the present pattern.

In many hospitals investigations continue to be poorly planned. Though entitled to practise what they believe to be right, clinicians could pay more heed to publications showing how admissions can be cut short by quicker mobilization of patients or by changing surgical procedure. Too often the work expands to fill the beds available. Waiting lists are not always the result of high demand and low facilities: they may be a sign of pride, or inefficiency.

If action was taken on all the above points it might, in many areas, allow a statutory cut in the allocation of beds. Where pressure is great this need not be proposed, but in most areas beds could be cut by between 10 and $25 \%$.

\section{Non-clinical Savings}

A strong stimulus to devolve patient care would be a policy (temporary and selective) of non-replacement of staff who resign or retire. This most certainly would arrest the growth of the consultant ranks and reduce the reliance on immigrant doctors. The cost of extra duty payments could be removed almost completely in an efficient hospital service. Projects whose efficacy is not certain, such as the N.H.S. Occupational Health Service, should not now be introduced in new areas. During the recent work to rule of ambulance staff the surprising mobility of many outpatients showed how much the service was being misused. Planners do get things wrong; just as Maplin was rendered unnecessary by an unexpected decline in air traffic so also can large parts of the hospital development programme be reviewed and postponed indefinitely. It is also inconceivable that nothing should be done to reverse the exponential demand for laboratory tests.

Capital investment need not be banned if rapid returns can be foreseen. The upgrading of outpatient departments and the introduction of progressive care are possible examples. Money spent improving records systems and communications is money saved in medical time and bed occupancy. Outside the hospital, delays and cancellations in building health centres have inhibited the primary care sector from growing in scope. This money must be found now, if need be from the local authorities-and would go almost unnoticed as a single $1-2 \%$ loading on the rates.

\section{General Practice}

The necessary changes in general practice are the corollary of those proposed for the hospital service. Supported by nurses and social services, well-trained doctors, combining individual interests with general family medicine in well-equipped health centres, can cut the pressure on the district hospital. Some also believe that family doctors should operate community hospitals, and there is general agreement that properly qualified doctors can conduct outpatient sessions in various specialties. General practitioners should organize themselves so that they dispense with the night deputizing services, which are one source of hospital overuse.

No short-term saving can be expected from developments in the field of preventive medicine, and detailed planning of future programmes must proceed. Savings from the results of health education and early diagnostic services seem the most promising areas.

\section{Administration}

So far little optimism can be felt that solutions to present day problems will come from changes in administration. All the specialist posts in community medicine are going to former regional board and local authority doctors who are founder Fellows and members of the new Faculty. Certainly many of these are excellent, but surely doctors from these backgrounds have no monopoly of the ideas needed to salvage the N.H.S. during the present economic drought. Indeed, the selection procedures, along with incomprehensible delays, have meant that most community physicians have spent most of 1974 in a state of personal professional concern while all the impetus of reorganization was lost. The advisory services look to be forming along hierarchical lines and, influenced by traditional consultants and family doctors, appear to be reshaping the pattern of the old N.H.S. within the new administrative framework. Administration at area and district level can gain respect and co-operation by itself reducing expenditure. But real co-operation from clinical staff will come only when proposed economies can be seen to be part of a coherent, well-publicized national plan.

The enthusiasm and initiative needed to bring about economy cannot be expected from staff who, at all levels, have been poorly paid for years. But staff cuts should make funds available, and the redistribution of these to those remaining (including junior doctors losing their extra duty payments) could be seen as productivity allowances. In fact, as largely untaxed money will be redistributed as taxable pay increases, probably $10 \%$ staff cuts could allow $20 \%$ increases for those remaining without any further cost to the taxpayer. It should be perfectly clear to everyone that people must be paid better if they are to co-operate in the type of changes I have suggested.

\section{Conclusion}

The problem of the N.H.S. is that its financial difficulties cannot be solved by individuals taking individual action, yet little seems likely to emerge from the essentially political confrontations between Government and health professions, and the professions and other N.H.S. employees. The N.H.S. is too unwieldy, hierarchical, and administratively constipated for much effective economy to take place. So probably services will decline and the strain will be taken off as private alternatives begin to flourish.

Bridge of Earn Hospital, Bridge of Earn, Perth

R. A. WOOD, B.SC., M.R.C.P., Consultant Physician

\section{Restricted List of National Health Service Drugs?}

\section{Noel D. L. Olsen}

In 1972-3 pharmaceutical services ( $£ 226$ million) and the hospital drug bill ( $£ 33_{4}^{3}$ million) came to a massive $£ 260$ million or $10 \%$ of the total cost of the Health Service, $(80 \%$ of this money was paid to manufacturers.) Most of this money is well spent, but some is wasted and enormous savings could be made. Doctors are rightly most resistant to any change that restricts their traditional clinical freedom, but clinical freedom should not be used as an excuse to cover incompetence or ignorance. Nevertheless, in the present economic situation, we must review all spending and make cuts wherever reasonable in the hope that money saved in one area might be used to better effect elsewhere.

While few doctors would support the limitation of prescribing to a government-controlled list of drugs-which would inevitably be inflexible and slow to change-I believe that voluntary limitation to 
those drugs which are included in the British National Formulary would be acceptable to the majority, and would still give each individual doctor the right to prescribe as he felt best in special situations. Furthermore, such limitation would actually improve the overall standard of prescribing especially if the advice given in the first half of the B.N.F. was observed. The present machinery, whereby the cost of a doctor's prescribing is kept under review and he is "contacted" if it becomes excessive, could be modified to pay special attention to the non-B.N.F. component as well as to the total cost. In 19723,035 "contacts" were made by regional medical officers as regards doctors prescribing. Only on one occasion was further action taken and eventually a small deduction was made from the doctor's remuneration because of excessive prescribing. In 19732,987 contacts were made but it appears that no further action was taken. I cannot believe that this one case was the only example of persistent overprescribing in two years and I feel that, to be effective, action must be taken far more frequently.

A few years ago two expert panels assessed all the drugs in M.I.M.S. and came to the conclusion that $35 \%$ of the preparations listed were undesirable. While the situation may have improved since, perhaps the time has now come for the D.H.S.S. to refuse to accept financial responsibility for the cost of prescriptions for preparations which are widely held to be undesirable (subject, of course, to reasonable appeal). Such a course would be infinitely preferable to a selective list of prescribable drugs, which would seriously affect the pharmaceutical industry. A strong and reasonably profitable industry is desirable both for future research and also to maintain exports (trade surplus-exports over imports-in 1973 was $£ 154$ million).

\section{Economy and Efficiency}

It is difficult to assess how much money would be saved by these measures, but they should tend to improve the overall standard of prescribing. Certainly far greater economy could be achieved if doctors were more critical of their prescribing and reassessed the need for each prescription carefully instead of just ordering a "repeat" when the patient reattends. (Evidence for overprescribing includes the results of collections of unwanted drugs -1 tons were collected a few years ago in one week in S. Wales.) Too often a p.r.n. prescription for night sedation is given as part of the hospital admission procedure, thus initiating an undesirable addiction. Bronchitics with irreversible airways obstruction frequently receive a useless prescription for bronchodilators when forceful advice about smoking would be more appropriate. Sore throats are often due to a viral infection but, when antibiotics are considered necessary, relatively expensive drugs, such as ampicillin, are often prescribed in place of the therapeutically more desirable drug of choice phenoxymethylpenicillin. Numerous other examples could be mentioned especially in relation to tranquillizers, antidepressants, antirheumatics, and antibiotics where a doctor often appears to be biased towards a particular preparation as a result of advertising rather than consideration of the results of scientific and clinical evaluation.

To conclude I propose that doctors be asked to voluntarily limit their prescribing to drugs recommended in the British National Formulary and that sanctions be more vigorously applied to restrain those who, in the view of their colleagues, regularly overprescribe. If we do not organize this inside the profession, more stringent restrictions may eventually be imposed from outside.

NOEL D. L. OLSEN, M.B., M.R.C.P., Medical Registrar

\section{Do All our Clinicopathological Investigations Pay Off?}

\section{N. K. Shinton}

Like most procedures in medicine, financial evaluation or costeffectiveness of clinicopathological investigations is almost impossible owing to variables such as time saved in hospital beds, time off work by patients, or psychological benefit to the patient-all of which may be influenced by factors other than the results of laboratory investigations. Hence the economic assessment of laboratory procedures is limited to studying costs of staff and equipment in relation to work load. Unfortunately the methods at present employed by the D.H.S.S. for correlating work load with staff are based on the number of requests and tests without any consideration of the enormous differences in the time and technical experience required from one test to another. In haematology the test time may vary from one minute for an erythrocyte sedimentation rate to 120 minutes for a red-cell enzyme assay; manual procedures taking nine minutes can be processed with print-out of results by an automated machine in one. A system of unit values for each test therefore has much to commend it.

Excluding capital development, present laboratory running costs are $80 \%$ for salaries and $20 \%$ for maintenance and replacement of equipment. So if there are to be effective economies in the laboratory service of the N.H.S. the staff/work load ratio must be improved by increasing the availability of equipment able to handle large work loads. Unfortunately the N.H.S. has a long way to go: about 500 laboratories are carrying out haematological tests but 300 of these perform less than 30,000 requests per annum (120 per day). Thus in most haematology laboratories expensive automated equipment would not be cost-effective. On the other hand, many of these laboratories are necessary to cover essential emergency procedures.

\section{Rationalization}

The staff/work load ratio could be improved for manual procedures by limiting the number of laboratories where each test is performed. For many procedures it takes little longer to perform 10 tests than a single one, and this applies similarly to clinical chemistry and to a lesser extent to microbiology. If N.H.S. finances are to be restricted the policy that every district general hospital should have a laboratory offering a complete range of tests cannot be maintained. There are two alternatives: to continue with these laboratories-limiting the number, range, and quality of the tests-or to rationalize the work among the various N.H.S. laboratories. Asking clinicians faced with a rising population of patients to reduce the load on the laboratory is not possible, and reduction in quality means wasted effort. Hence a reduction in the range of routine tests offered by each laboratory is the only solution.

The D.H.S.S. has made a start in rationalization by setting up supraregional laboratories for some procedures. Rationalization is unpopular with both laboratory and clinical staff because inevitably it leads to lack of personal communication, delay in transport of specimens and return of reports, and a lessened attractiveness of a laboratory for training. To some extent these drawbacks can be avoided by preliminary explanation, improved transport, the installation of data processing, and integration of training programmes among laboratories. Unless N.H.S. laboratories become more efficient economically it is unlikely that money will be available in the near future for new developments-and without these interest in medicine will decline and standards of practice fall.

\section{Department of Haematology, Coventry and Warwickshire Hospital,} Coventry CV1 4FH

N. K. SHINTON, M.R.C.PATH., F.R.C.P., Consultant Haematologist

\section{Can We Audit Cost-effectively?}

\section{Hugh Dudley}

I am worried both about the title and the implications of my subject. The title suggests the excessively transatlantic jargon of the economist, though .I am relieved to see in the Oxford English Dictionary that one of the first uses of audit was more general, as a "searching examination or solemn rendering of accounts especially the Day of Judgement". Cost-effective is a Johnny-come-lately but has reached the O.E.D. Supplement, probably as a result of Mr. McNamara's stay at the Pentagon in the mid-sixties. If I were patient enough to search through the economic literature I could probably find it well defined, but for me it means that if we spend a penny on analysing what we do we get a penny or more back in the good that accrues to the community. And there's the rub, because, if Mr. McNamara's analysis of the cost of the F 1-11 fighter-bomber versus its strike 